\title{
pH sensor based on tilted fiber Bragg gratings covered by a sol-gel
}

\author{
Marc Debliquy ${ }^{1}$, Jean-Michel Renoirt ${ }^{1,2}$, Christophe Caucheteur ${ }^{2}$, Patrice Mégret ${ }^{2}$ \\ and Marie-Georges Olivier ${ }^{1}$, \\ ${ }^{1}$ Material Science Department, University of Mons, rue de l'Epargne 56, B-7000 Mons \\ ${ }^{2}$ Electromagnetism and Telecommunications Department, University of Mons, Boulevard Dolez 31, B- \\ 7000 Mons \\ marc.debliquy@umons.ac.be
}

\begin{abstract}
This paper presents the development of a $\mathrm{pH}$ sensor based on a Tilted Fiber Bragg Grating (TFBG) covered with a microporous coating consisting of bromophenol blue ( $\mathrm{pH}$ indicator) encapsulated in a silica sol-gel matrix. The covered TFBG shows important modifications of its transmission amplitude spectrum in the range of $1510-1590 \mathrm{~nm}$ as a function of the $\mathrm{pH}$. All the cladding mode resonances show a peak-to-peak amplitude decrease with increasing $\mathrm{pH}$ according to a sigmoidal evolution. The response, defined as the change in amplitude of a resonance peak as a function of the $\mathrm{pH}$, is fast $(<10 \mathrm{~s})$ and reversible. The amplitude change between acidic and basic forms is about $4 \mathrm{~dB}$ at 1550 $\mathrm{nm}$. This kind of sensor could be of interest for practical applications such as corrosion monitoring.
\end{abstract}

Key words: pH sensor, sol-gel, optical fiber, TFBG, Tilted Bragg Gratings.

\section{Introduction}

During the last decades, there has been a rising interest in the development of sensors based on optical fibers for many different applications, including chemical and gas sensing [1,2]. Numerous transduction techniques were studied and fiber Bragg gratings (FBGs) appeared to be very attractive candidates [3-5]. An FBG is a periodic and permanent modulation of the core refractive index of an optical fiber along the propagation axis. It is generally obtained by exposing a photosensitive optical fiber to an intense UV interference pattern. A fiber Bragg grating acts as a wavelength selective filter around the socalled Bragg wavelength [6]. The period $\Lambda$ is usely around $500 \mathrm{~nm}$ and the Bragg wavelength is around $1550 \mathrm{~nm}$.

Tilted Fiber Bragg Gratings (TFBG) present a refractive index modulation angled by a few degrees relative to the perpendicular to the optical fiber axis. They couple light between the core and the cladding. As a result, they present a comb-like transmission amplitude spectrum comprising several tens of resonances, each one characterized by its own sensitivity to the surrounding refractive index. The important point is that the position and the amplitude of these peaks depend on the refractive index of the surrounding medium. When immersed in a medium with a refractive index that matches the effective refractive index of some cladding modes, these modes become weakly guided, and the narrow resonance peaks are replaced by a smooth loss continuum.

TFBGs naturally yield refractometers accurate to $10^{-4}$ RIU (refractive index unit) [7-10]. More recently, TFBGs were used for sensing chemical or biochemical quantities [11].

TFBGs used as sensors present decisive advantages like small size (which is compatible with the use of tiny sample volumes), electromagnetic interferences immunity and high accuracy. To achieve these performances, various demodulating methods have been reported, based on whole spectrum analysis [7, 8], on single resonance tracking [12] or transmission power measurement [13]. Using wavelength encryption of the signal allows temperature-insensitive measurements [14].

Specific chemical sensors can be built by using TFBGs covered with a dedicated coating that changes its refractive index when in contact with target chemical species. Doing so, the sensors inherit the above-mentioned advantages of the optical fiber sensors.

This study demonstrates the possibility to sense $\mathrm{pH}$ variations with the help of a microporous 
sol-gel coating encapsulating a $\mathrm{pH}$ indicator whose refractive index changes due to the modification of the indicator state. Silica sol-gel was chosen because of its refractive index close to that of the fiber, enhancing in turn the response. Bromophenol blue was used due to its high Kreft's dichromaticity index and its convenient $\mathrm{pKa}$.

In comparison to other existing optical sensors $[15,16]$, using TFBGs brings several benefits. They preserve the fiber integrity while offering intrinsic temperature insensitivity. Also, measurements can be operated at standard telecommunication wavelengths.

\section{Sample preparation}

Experiments were carried out on TFBGs classically manufactured into hydrogen-loaded single mode optical fiber (germanium doped core) by means of a $1095 \mathrm{~nm}$ uniform phase mask and a frequency-doubled Argon-ion laser emitting at $244 \mathrm{~nm}$. The phase mask was tilted in the plane perpendicular to the incident beam. An external tilt angle of $6^{\circ}$ was chosen. The protection coating was mechanically removed before inscription. After that, and before coating, gratings were annealed at $80{ }^{\circ} \mathrm{C}$ during 12 hours in air to remove the hydrogen and stabilize their physical properties.

The sensitive sol-gel coating was obtained by incorporation of the $\mathrm{pH}$ indicator, here bromophenol blue, in an alcoholic solution (15 $\mathrm{ml})$ containing TEOS as precursor $(15 \mathrm{ml})$, water $(3 \mathrm{ml})$ and $\mathrm{HCl}$ as catalyst $(2 \mathrm{M} 0.25 \mathrm{ml})$. The solution was heated at $60{ }^{\circ} \mathrm{C}$ (reflux) for $1 \mathrm{~h}$ and aged for 1 week before use.

The sensitive layer was deposited on the fiber by dip coating in one step and annealed at 80 ${ }^{\circ} \mathrm{C}$ for 1 hour, yielding a $5 \mu \mathrm{m}$ thick transparent yellowish layer.

\section{Experimental setup}

The amplitude transmission spectra were measured using an ASE (amplified stimulated emission) optical source (Ammonics) and an optical spectrum analyzer Ando AQ6317C (figure 1). The fibers were immersed in a $1 \mathrm{M}$ $\mathrm{NaCl}$ solution and the $\mathrm{pH}$ was adjusted by adding $1 \mathrm{M} \mathrm{HCl}$ and $\mathrm{NaOH}$ solution drops. The $\mathrm{pH}$ was controlled with a glass electrode pHmeter (Metrohm). $\mathrm{NaCl}$ is introduced to ensure that the refractive index of the solution is constant.

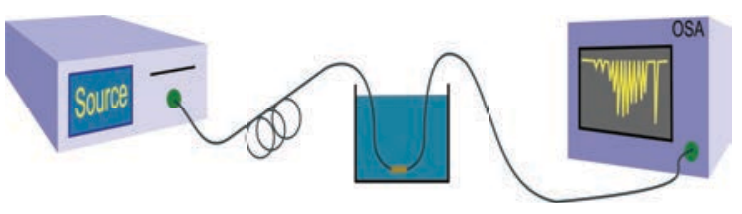

Fig. 1: Experimental setup.
The sweeping time for $10 \mathrm{pm}$ resolution in the whole range $(1520-1590 \mathrm{~nm})$ is about $40 \mathrm{~s}$.

\section{Results and discussion}

Figure 2 displays, with an offset in the vertical axis, the TFBG transmitted amplitude spectra for various $\mathrm{pH}$ values. The resonance modes are drastically affected by the $\mathrm{pH}$ variations (figure 3). This results from the fact that, for solutions having $\mathrm{pH}$ lower than 5 (acidic form of the indicator), the coating containing the indicator is yellow and for $\mathrm{pH}>6$ (basic form), it becomes dark blue. For a free indicator, this color change is observed in the $\mathrm{pH}$ range 3-4.6. In addition, the refractive index of the coating increases when the indicator is in its blue form.

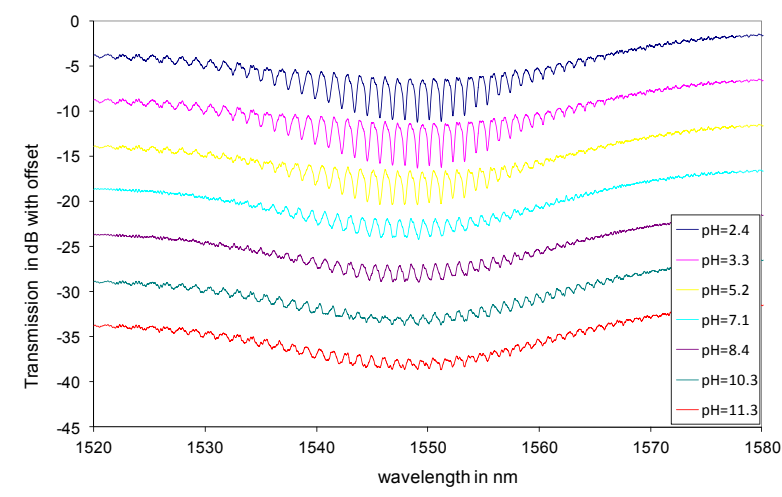

Fig. 2: Transmission spectrum of the TFBG at different $\mathrm{pH}$ values.

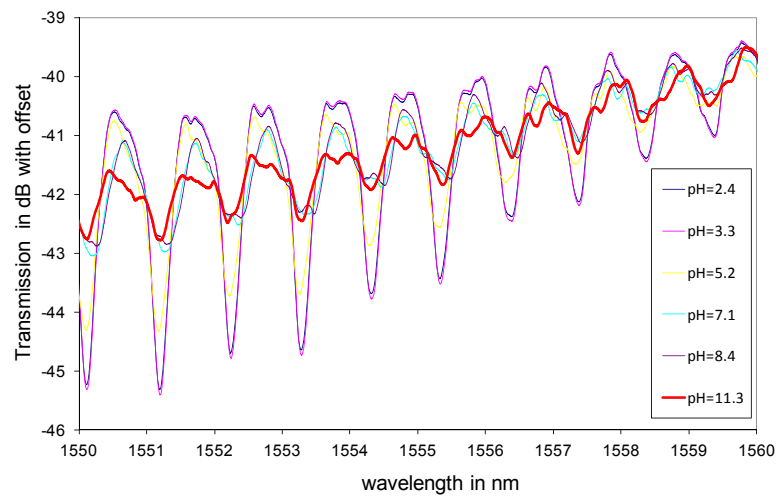

Fig. 3: Transmission spectrum of the TFBG at different $\mathrm{pH}$ values. Zoom around $1555 \mathrm{~nm}$.

A simple way of demodulating the amplitude spectrum is to track the peak-to-peak amplitude of a given cladding mode resonance as a function of the $\mathrm{pH}$. Figure 4 shows the amplitude evolution versus $\mathrm{pH}$ for the peak centered around $1550 \mathrm{~nm}$.

As could be expected, the response of the sensor presents a reversible sigmoidal shape.

When following one given peak, the scan can be refreshed each second allowing to measure the response time. The response time is evaluated to about 10 s when going from $\mathrm{pH} 3$ to $\mathrm{pH} 10$ or vice versa. 


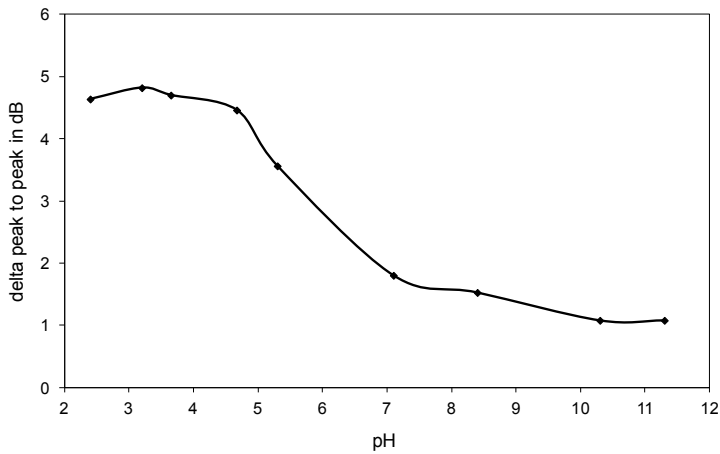

Fig. 4: Peak-to-peak amplitude of the cladding mode resonance near $1550 \mathrm{~nm}$ at different $\mathrm{pH}$ values.

\section{Conclusions}

TFBGs covered by a sol-gel layer show important modifications of their transmission amplitude spectrum in the range $1510-1590 \mathrm{~nm}$ as a function of the $\mathrm{pH}$. All the cladding mode resonances present a peak-to-peak amplitude decrease with increasing $\mathrm{pH}$ values, according to a sigmoidal evolution. The amplitude change between acidic and basic forms is about $4 \mathrm{~dB}$ at $1550 \mathrm{~nm}$. The response, defined as the change in the amplitude of a resonance peak, is fast ( 10s) and reversible.

This kind of sensor could be of interest for practical applications such as corrosion monitoring.

\section{Acknowledgements}

This study was performed in the framework of the Opti ${ }^{2}$ Mat project financially supported by the Walloon region in Belgium. C. Caucheteur is supported by the Fonds National de la Recherche Scientifique (F.R.S.-FNRS).

\section{References}

[1] B. Lee, "Review of the present status of optical fiber sensors" Opt. Fiber Technol. 9, 57-79 (2003); doi: 10.1364/JOSAB.25.000310

[2] Culshaw B., "Research to reality: bringing fibre optic sensors into applications", Proc. of SPIE, 5952, No.01, 01-15, (2005);doi:10.1117/12

[3] C. Elosua, I. R. Matias, C. Bariain and F. J. Arregui, "Volatile Organic Compound Optical Fiber Sensors: A Review" Sensors 6, 1440-1465 (2006); doi:10.3390/s6111440

[4] M. El-Sherif, L. Bansal, and J. Yuan, "Fiber optic sensors for detection of toxic and biological threats," Sensors 7(12), 3100-3118 (2007); doi:10.3390/s7123100.

[5] C. Caucheteur, M. Debliquy, D. Lahem, P. Mégret, "Hybrid fiber gratings coated with a catalytic sensitive layer for hydrogen sensing in air", Optics Express, 16, n. 21, 16854-16859 (2008); doi:10.1364/OE.16.016854

[6] A.D.Kersey, M.A.Davis, H.J.Patrick, M. LeBlanc, K.P. Koo, C.G. Askins, M.A. Putnam, E. J. Friebele, "Fiber Bragg grating sensors", IEEE Journal of Lightwave Technology 15, 1442-1463 (1997); doi: 10.1109/50.618377

[7] G. Laffont, P. Ferdinand, "Tilted short-period fibre-Bragg-grating-induced coupling to cladding modes for accurate refractometry," Meas. Sci. Technol. 12, 765-770 (2001); doi: 10.1364/OE.18.000713

[8] C. Caucheteur, P. Megret, "Demodulation technique for weakly tilted fiber Bragg grating refractometer," Photon. Technol. Lett. 17, 27032705 (2005); doi: 10.1109/LPT.2005.859411

[9] C.-F. Chan, C. Chen, A. Jafari, A. Laronche, D. J. Thomson, J. Albert, "Optical fiber refractometer using narrowband cladding-mode resonance shifts," Appl. Opt. 46, 1142-1149 (2007); doi: 10.1364/AO.46.001142

[10] J. Albert, L.-Y. Shao, and C. Caucheteur, "Tilted fiber Bragg gratings sensors," Laser \& Photonics Reviews 26, 22 (2012); doi:10.1002/lpor.201100039

[11] F. Baldini, M. Brenci, F. Chiavaioli, A. Giannetti, and $\mathrm{C}$. Trono, "Optical fibre gratings as tools for chemical and biochemical sensing," Analytical and bioanalytical chemistry 402, 109-116 (2011); doi: $10.1007 / \mathrm{s} 00216-011-5492-3$

[12] C. Chen, T. Guo, A. Laronche, and J. Albert, "Radiation mode resonances of tilted fiber Bragg gratings for high index media measurement," Proceedings of SPIE 7004, 700418-1-700418-4, Spie (2008); doi:10.1117/12.785661

[13] Y. Miao, B. Liu, and Q. Zhao, "Refractive index sensor based on measuring the transmission power of tilted fiber Bragg grating," Optical Fiber Technology 15, 233-236 (2009). doi: 10.1016/j.yofte.2008.11.002

[14] H. De-bo and J. Qi, "Thermal Independent Solution Concentration Sensing with Tilted Fiber Bragg Grating," Library 345, 3-8 (2010).

[15] S. Dong, M. Luo, G. Peng, W. Cheng, "Broad range $\mathrm{pH}$ sensor based on sol-gel entrapped indicators on fibre optic", Sensors and Actuators B: Chemical, 129, pp 94-98 (2008); doi:10.1016/j.snb.2007.07.078

[16] B.D. Gupta, D.K. Sharma, "Evanescent wave absorption based fiber optic $\mathrm{pH}$ sensor prepared by dye doped sol-gel immobilization technique", Optics Communications 140, 32-35 (1997); doi:10.1016/S0030-4018(97)00162-4 